\title{
SPI- I and SPI-2 defective mutants of salmonella enterica serovar enteritidis: promising future salmonella vaccine for poultry
}

\author{
Keywords: salmonella vaccine, poultry, SPI-1 and SPI-2 mutants
}

Abbreviations: SPI, salmonella pathogenicity island; T3SS, type iii secretion system; fliC, gene encoding for flagella; lon, gene coding for lon protease

\section{Editorial}

Non-typhoid Salmonella enterica serovars are among the most common causative agents of food-borne diseases in human worldwide. ${ }^{1}$ Since poultry is the most frequent reservoir of salmonellosis for humans, vaccination of chickens is considered as an effective measure to decrease $S$. enterica prevalence in poultry as well as to decrease $S$. enterica incidence in humans. Within the last 25years, many live $S$. enterica vaccines have been described and those with inactivated aro or phoP genes were amongst the first ones tested., Currently, construction of attenuated vaccine strains of $S$. enterica is not an issue and many different mutants have been tested in mice, chickens and even humans. ${ }^{4-7}$ However, the main dilemma is which mode of attenuation to choose out of the many possibilities. The major pathogenicity islands of $S$. enterica include SPI-1, SPI-2, SPI- 3, SPI4 and SPI-5. The SPI-1 and SPI-2 genes code for proteins forming the type III secretion system (T3SS) which enable the transport of $S$. enterica proteins from the bacterial cell directly into the cytosol of eukaryotic cells. The SPI-1 encoded T3SS is required for the transport of $S$. enterica proteins across the cytoplasmic membrane of a host cell into its cytosol where they induce cytoskeletal rearrangements resulting in the uptake of $S$. enterica even by non-phagocytic cells. ${ }^{8}$ In addition, it has been reported that SPI-1 genes, independent of cell invasion, induce macrophage cytotoxicity. ${ }^{9}$ SPI-2 encoded T3SS is required for the transport of $S$. enterica proteins across the phagosomal membrane and increases $S$. enterica survival inside phagocytic cells. ${ }^{10,11}$ The function of genes localized on the remaining SPIs is less well characterized and according to recent reports these remaining SPIs individually have no effect on S. Enteritidis virulence although collectively they have a low effect on bacterial colonization. ${ }^{7}$

Recently, with an increasing understanding of $S$. enterica pathogenesis, mutants without a functional type III secretion system (T3SS) encoded by either SPI-1 or SPI-2 have been tested for determining their virulence and vaccine potentials. Results of such studies show that whilst SPI2 mutants of S. enterica are attenuated in all warm-blooded hosts, SPI1 mutants seem to be attenuated only in hosts for which an enteric type of disease is characteristic and these genes are dispensable when the output of the infection is a typhoid disease. ${ }^{12-14}$ In agreement with the previous statement, the removal of SPI1 genes from S. Enteritidis or S. Typhimurium, i.e. the serovars which cause a mild enteric disease in chickens, results in a decrease in virulence with preserved immunogenicity in these hosts. ${ }^{7,15,16}$ Moreover, SPI1 mutants are defective in early interactions with macrophages which may enable the macrophage's proper
Volume 3 Issue 2 - 2016

\author{
Md. Masudur Rahman \\ Department of Pathology, Sylhet Agricultural University, \\ Bangladesh
}

\begin{abstract}
Correspondence: Md. Masudur Rahman, Department of Pathology, Faculty of Veterinary and Animal Science, Sylhet Agricultural University, Sylhet 3100, Bangladesh, Tel +880821761002(201), Fax +880821761980, Email rahmanmm.dpp@sau.ac.bd
\end{abstract}

Received: January 29, 2016 | Published: February 24, 2016

antigen processing and presentation ${ }^{17-19}$ though the role of SPI1 in the interactions with other antigen presenting cells in the chicken is less clear. When SPI1 and SPI2 mutants of S. enterica serovar Enteritidis have been tested for their vaccine potentials in chickens, both the mutants provide protection to chicken against S. Enteritidis challenge as documented by findings such as the bacterial counts in tissues, spleen weight, antibody production and cytokine response (namely IL-17 and IL-22). When the 2 mutants are compared, vaccination with the SPI1 mutant proved to be more effective in the protection of chicken against $\mathrm{S}$. Enteritidis challenge than the vaccination with the SPI2 mutant. On the other hand, vaccination with the SPI2 mutant stimulates a slightly higher antibody production and such a mutant might therefore be a better choice if Salmonella is used as a vector for the delivery of heterologous antigens with a desired stimulation of the humoral part of the immune system. ${ }^{15}$ Recently, a triple SPI1lon-fliC mutant of S. Enteritidis has been constructed and tested for its efficacy as a live attenuated marker vaccine for the oral vaccination of poultry. ${ }^{20}$ Deletion of fliC gene encoding for flagella in this mutant strain enables serological differentiation of vaccinated and infected chickens which is an increasing demand for the days and is something that the current commercial vaccines cannot provide. Lon protease is a negative regulator of SPI1 genes ${ }^{21}$ and is required for the resistance to multiple environmental stresses ${ }^{22}$ and removal of lon reduces the virulence of S. Enteritidis even for highly sensitive Balb/C mice. ${ }^{13}$ Therefore, the inactivation of gene encoding Lon protease results in further independent attenuation of S. Enteritidis in virulence with a mucoid colony phenotype due to the overproduction of capsular polysaccharides that enables additional simple differentiation of the vaccine strain from those circulating in the environment. The SPI1lon-fliC mutant might therefore be a suitable marker vaccine strain for oral vaccination of poultry in future.

\section{Acknowledgements}

The author thanks Dr. Ivan Rychlik, Veterinary Research Institute, Brno, Czech Republic for sharing his research articles. 


\section{Conflict of interest}

The author declares no conflict of interest.

\section{References}

1. Majowicz SE, Musto J, Scallan E, et al. The global burden of nontyphoidal Salmonella gastroenteritis. Clin Infect Dis. 2010;50(6):882-889.

2. Cooper GL, Nicholas RA, Cullen GA, et al. Vaccination of chickens with a Salmonella enteritidis aroA live oral Salmonella vaccine. Microb Pathog. 1990;9(4):255-265.

3. Groisman EA, Parra-Lopez C, Salcedo M, et al. Resistance to host antimicrobial peptides is necessary for Salmonella virulence. Proc Natl Acad Sci USA. 1992;89(24):11939-11943.

4. Hoiseth SK, Stocker BAD. Aromatic-dependent Salmonella typhimurium are non-virulent and effective as live vaccines. Nature. 1981;291(5812):238-239.

5. Galan JE, Curtiss R III. Virulence and vaccine potential of phoP mutants of Salmonella typhimurium. Microb Pathog. 1989;6(6):433-443.

6. Bohez L, Ducatelle R, Pasmans F, et al. Long-term colonisationinhibition studies to protect broilers against colonisation with Salmonella Enteritidis, using Salmonella Pathogenicity Island 1 and 2 mutants. Vaccine. 2007;25(21):4235-4243.

7. Rychlik I, Karasova D, Sebkova A, at al. Virulence potential of five major pathogenicity islands (SPI-1 to SPI-5) of Salmonella enterica serovar Enteritidis for chickens. BMC Microbiol. 2009;9:268.

8. Kaniga K, Trollinger D, Galan JE. Identification of two targets of the type III protein secretion system encoded by the inv and spa loci of Salmonella typhimurium that have homology to the Shigella IpaD and IpaA proteins. J Bacteriol. 1995;177(24):7078-7085.

9. Chen LM, Kaniga K, Galan JE. Salmonella spp. are cytotoxic for cultured macrophages. Mol Microbiol. 1996;21(5):1101-1115.

10. Cirillo DM, Valdivia RH, Monack DM, et al. Macrophagedependent induction of the Salmonella pathogenicity island 2 type III secretion system and its role in intracellular survival. Mol Microbiol. 1998;30(1):175-188.

11. Hensel M, Shea JE, Waterman SR, et al. Genes encoding putative effector proteins of the type III secretion system of Salmonella pathogenicity island 2 are required for bacterial virulence and proliferation in macrophages. Mol Microbiol. 1998;30(1):163-174.
12. Jones MA, Wigley P, Page KL, et al. Salmonella enterica serovar Gallinarum requires the Salmonella pathogenicity island 2 type III secretion system but not the Salmonella pathogenicity island 1 type III secretion system for virulence in chickens. Infect Immun. 2001;69(9):5471-5476.

13. Karasova D, Sebkova A, Vrbas V, et al. Comparative analysis of Salmonella enterica serovar Enteritidis mutants with a vaccine potential. Vaccine. 2009;27(38):5265-5270.

14. Murray RA, Lee CA. Invasion genes are not required for Salmonella enterica serovar typhimurium to breach the intestinal epithelium: evidence that Salmonella Pathogenicity Island 1 has alternative functions during infection. Infect Immun. 2000;68(9):5050-5055.

15. Matulova M, Havlickova H, Sisak F, et al. Vaccination of chickens with Salmonella Pathogenicity Island (SPI) 1 and SPI2 defective mutants of Salmonella enterica serovar Enteritidis. Vaccine. 2012;30(12):20902097.

16. Dieye Y, Ameiss K, Mellata M, et al. The Salmonella Pathogenicity Island (SPI) 1 contributes more than SPI2 to the colonization of the chicken by Salmonella enterica serovar Typhimurium. BMC Microbiol. 2009;9:3.

17. Monack DM, Raupach B, Hromockyj AE, et al. Salmonella typhimurium invasion induces apoptosis in infected macrophages. Proc Natl Acad Sci USA. 1996;93(18):9833-9838.

18. Pavlova B, Volf J, Ondrackova P, et al. SPI-1- encoded type III secretion system of Salmonella enterica is required for the suppression of porcine alveolar macrophage cytokine expression. Vet Res. 2011;42:16.

19. Pullinger GD, Paulin SM, Charleston B, et al. Systemic translocation of Salmonella enterica serovar Dublin in cattle occurs predominantly via efferent lymphatics in a cell-free niche and requires type III secretion system 1 (T3SS-1) but not T3SS-2. Infect Immun. 2007;75:5191-5199.

20. Matulova M, Havlickova H, Sisak F, et al. Vaccination of Chickens with SPI1-lon and SPI1-lon-fliC Mutant of Salmonella enterica Serovar Enteritidis. PLoS ONE. 2013;8(6):e66172.

21. Takaya A, Tomoyasu T, Tokumitsu A, et al. The ATP-dependent lon protease of Salmonella enterica serovar Typhimurium regulates invasion and expression of genes carried on Salmonella Pathogenicity Island $1 . J$ Bacteriol. 2002;184(1):224-232.

22. Leyman B, Boyen F, Van Parys A, et al. Tackling the issue of environmental survival of live Salmonella Typhimurium vaccines: deletion of the lon gene. Res Vet Sci. 2012;93(3):1168-1172. 